\title{
Relativistic Calculations for Photonuclear Reactions (II). Nonrelativistic reductions and nuclear medium effects*
}

\author{
M. Hedayati-Poor, J.I. Johansson and H.S. Sherif \\ Department of Physics, University of Alberta \\ Edmonton, Alberta, Canada T6G 2J1
}

May 4, 2018

\begin{abstract}
The relativistic amplitude for the direct knock-out contribution to $(\gamma, p)$ reactions on nuclei is reduced to a nonrelativistic form using an effective Pauli reduction scheme. The reduction is carried out to second order in the inverse nucleon mass. It is found that the interaction Hamiltonian appearing in the nonrelativistic amplitude has significant dependence, starting at second order, on the vector and scalar mean nuclear potentials. These strong medium modifications are absent in traditional nonrelativistic calculations. Detailed comparisons show that these modifications are crucial to understanding the differences between relativistic and nonrelativistic models. These differences are also examined through reduction of the relativistic amplitude via the Foldy-Wouthuysen transformation. Similar medium modifications are obtained in this case as well. We discuss the implications of these medium modifications for the consistency of existing nonrelativistic calculations.
\end{abstract}

\footnotetext{
*Work supported in part by the Natural Sciences and Engineering Research Council of Canada
} 


\section{Introduction}

Recently Lotz and Sherif have calculated the relativistic amplitude for the direct knock-out contribution to $(\gamma, p)$ reactions in a distorted wave approximation [1]. Similar calculations have also been given by McDermott et al. [2]. The results obtained from these relativistic calculations were found to be in better agreement with experimental data than those of nonrelativistic distorted wave Born approximation (DWBA) calculations [3]. The latter calculations are based on the use of Schrödinger wave functions to describe the bound and continuum nucleons. The nuclear current is obtained through a Foldy-Wouthuysen (FW) transformation of the relativistic Hamiltonian describing the interaction of a photon with a free nucleon 4 , 5].

In this paper we clarify the main differences between the relativistic and nonrelativistic calculations. We obtain the nonrelativistic amplitude from the relativistic distorted wave $\mathrm{S}$ matrix through two different reduction schemes. The first of these is the Pauli reduction in which the relativistic $\mathrm{S}$ matrix of the $(\gamma, p)$ reaction is expressed in a form that involves Schrödinger-like wave functions and an effective interaction Hamiltonian. This Hamiltonian is expanded in powers of $1 /(E+M)$, where $M$ is the nucleon mass and $E$ is its total energy. The first order terms in this expansion, in the limit $E \rightarrow M$, reproduce the form of the usual nonrelativistic amplitude. A characteristic feature of the higher order terms is the dependence of the interaction Hamiltonian on the nuclear vector and scalar potentials. A short account of this discussion has been published [6]. This scheme has also been applied to a similar study of the relativistic and nonrelativistic descriptions of $\left(e, e^{\prime} p\right)$ reactions [7].

The second approach is based on the use of the Foldy-Wouthuysen transformation. The relativistic knock-out amplitude is written for a model case in which the initial bound and final continuum nucleons are described by a single Dirac Hamiltonian with real vector and scalar potentials. Through the FW reduction we are able to write a nonrelativistic limit of the amplitude to various orders in the inverse nucleon mass. Again we find that the interaction Hamiltonian is dependent on the strong nuclear potentials.

We begin section 1 by summarizing the calculation of the relativistic $\mathrm{S}$ matrix describing the direct knock-out contribution to $(\gamma, p)$ reactions on nuclei. In addition we provide some discussion of the Dirac equation containing strong scalar and vector potentials. In section 2 we illustrate the formalism 
of the Pauli reduction and show how the relativistic $\mathrm{S}$ matrix of the $(\gamma, p)$ reaction is reduced to an expansion in the inverse nucleon mass. We then show results of detailed calculations and discuss the implications of the presence of the nuclear potentials in the interaction Hamiltonian. In section 3 we discuss the FW transformation of a relativistic Hamiltonian describing a particle interacting with electromagnetic and strong nuclear fields, and we find the corresponding nonrelativistic amplitude to second order in the inverse nucleon mass. We discuss differences between the interaction Hamiltonians obtained through the Pauli and FW reduction schemes. The section closes with a discussion of observables calculated at different orders, with and without potentials in the FW interaction Hamiltonian. Section 4 is devoted to conclusions.

\section{Relativistic Direct Knock-out Mechanism}

The relativistic distorted wave amplitude (S matrix) for the reaction $(\gamma, p)$ on a target nucleus in the angular momentum state $\left|J_{i} M_{i}\right\rangle$ leading to a residual nuclear state $\left|J_{f} M_{f}\right\rangle$ is obtained in first order in the interaction Hamiltonian as [1]

$$
\begin{aligned}
S_{f i}= & \frac{-i}{(2 \pi)^{3}}\left[\frac{1}{2 \omega}\right]^{1 / 2}\left[\frac{M}{E_{C}}\right]^{1 / 2} \\
& \times \sum_{J_{B} M_{B}}\left(J_{f}, J_{B} ; M_{f}, M_{B} \mid J_{i}, M_{i}\right)\left[\mathcal{S}_{J_{i} J_{f}}\left(J_{B}\right)\right]^{1 / 2} \\
& \times \int \bar{\psi}_{S_{f}}^{(-)}(x) H_{e m}(x) \psi_{J_{B} M_{B}}(x) d^{4} x
\end{aligned}
$$

where $E_{C}$ is the energy of the final state continuum proton and $M$ is its mass. The energy of the incident photon is $\omega$. The Clebsch-Gordan coefficient in equation (1) couples the bound proton with angular momentum $J_{B}$ and projection $M_{B}$ to the residual nucleus, and $\mathcal{S}$ is the spectroscopic factor. The electromagnetic interaction Hamiltonian is

$$
H_{e m}(x)=e A(x)+\frac{\kappa}{2} \frac{e}{2 M} \sigma^{\mu \nu} F_{\mu \nu}(x) .
$$

Notice that in this work we do not consider modifications to the electromagnetic interaction Hamiltonian arising when a nucleon is off-shell [8]. The 
4 -vector potential describing the photon is written as

$$
A_{\xi}^{\mu}(x)=\epsilon_{\xi}^{\mu} e^{-i k_{\gamma} \cdot x},
$$

where the label $\xi$ is the polarization angle of the photon, allowing us to choose between two linear polarization states when the polarization 4-vector is written as $\epsilon_{\xi}^{\mu}=(0, \cos \xi, \sin \xi, 0)$. In writing the polarization vector this way we have made a definite choice of z-axis; namely, the z-axis is taken along the direction of the photon momentum so the photon 4-momentum is always $k_{\gamma}^{\mu}=\left(E_{\gamma}, 0,0, k_{\gamma}\right)$. We thus have two linear polarization states: $\xi=0^{\circ}$ with polarization along the $\mathrm{x}$-axis and $\xi=90^{\circ}$ with polarization along the y-axis.

The second term in the interaction Hamiltonian (2) is an anomalous magnetic moment term with $\kappa=1.79$ for the proton. The tensor $\sigma^{\mu \nu}$ is related to the usual Dirac gamma matrices through,

$$
\sigma^{\mu \nu}=\frac{i}{2}\left[\gamma^{\mu}, \gamma^{\nu}\right]
$$

and the electromagnetic field tensor $F_{\mu \nu}$ is

$$
F_{\mu \nu}=\partial_{\mu} A_{\nu}-\partial_{\nu} A_{\mu} .
$$

The Dirac spinors in equation (1) describe the bound and continuum nucleons and are solutions of a Dirac equation of the form

$$
\{\boldsymbol{\alpha} \cdot \boldsymbol{p}+\beta[M+S(r)]+V(r)\} \psi(x)=E \psi(x),
$$

where we adopt the standard representation of the $4 \times 4$ Dirac matrices $\left\{\alpha_{i}\right\}$ and $\beta$ [9]. The potentials $S(r)$ and $V(r)$ are the scalar and zeroth-component vector potentials respectively. These potentials are originally derived from the effective Lagrangian of the $\sigma+\omega$ model [10]. In actual distorted wave calculations, the final state proton is described by a solution of the Dirac equation above, containing complex potentials $S_{C}(r)$ and $V_{C}(r)$. The parameters for these potentials are obtained through analysis of data from proton elastic scattering on nuclei [11]. For the bound nucleon, Dirac-Hartree potentials are used [12]. Detailed discussions of the Dirac equations pertaining to both the bound and continuum nucleons can be found in references [1, 12].

In the rest of this section we discuss a Schrödinger-like wave equation derived from the Dirac equation (6). The solutions of this Schrödinger-like equation will be used in the next section to cast the amplitude in a nonrelativistic form. 
We write the Dirac spinors in terms of upper and lower components $u$ and $\ell$

$$
\psi(x)=\left[\begin{array}{l}
u(x) \\
\ell(x)
\end{array}\right],
$$

and use the Dirac equation (6) to write the lower component of the wave function in terms of the upper component as

$$
\ell(x)=\frac{\boldsymbol{\sigma} \cdot \boldsymbol{p}}{E+M+S(r)-V(r)} u(x) .
$$

Thus the Dirac spinors can be written in terms of their upper components in the form

$$
\psi(x)=\left[\begin{array}{c}
1 \\
M+E+S(r)-V(r)
\end{array}\right] u(x) .
$$

The upper component of the Dirac spinor $u(x)$ can be related to a Schrödinger-like wave function $\Psi_{\text {Sch }}(x)$ by [13]

$$
u(x)=D^{\frac{1}{2}}(r) \Psi_{\mathrm{Sch}}(x),
$$

and the function $D(r)$ depends on the Dirac potentials as

$$
D(r)=\frac{E+M+S(r)-V(r)}{E+M}=\frac{s(r)}{E+M},
$$

Note that $D(r)$ goes to one for large $r$, so the asymptotic behavior of the upper component of the Dirac wave function and the Schrödinger-like wave function are the same. The Schrödinger-like wave function $\Psi_{\text {Sch }}(x)$ is the solution of the Schrödinger-like equation [13]

$$
\left\{-\frac{\boldsymbol{\nabla}^{2}}{2 M}+U_{\text {cent }}(r)+U_{\text {so }}(r) \boldsymbol{\sigma} \cdot \boldsymbol{L}\right\} \Psi_{\text {Sch }}=(E-M) \Psi_{\text {Sch }} .
$$

The central and spin-orbit potentials are functions of the Dirac potentials as well as the energy of the proton, and are written explicitly as

$$
U_{\text {cent }}(r)=E-M+\frac{1}{2 M}\left\{s d-\frac{s^{\prime}}{s r}-\frac{1}{2} \frac{s^{\prime \prime}}{s}+\frac{3}{4} \frac{s^{\prime 2}}{s^{2}}\right\},
$$


and

$$
U_{\mathrm{so}}=-\frac{1}{2 M} \frac{s^{\prime}(r)}{r s(r)}
$$

In addition to the function $s(r)$ defined in equation (11), we have defined a function involving the difference of the nucleon's mass and energy

$$
d(r)=M-E+S(r)+V(r) .
$$

For the bound state this "nonrelativistic" equation provides a better description of spin orbit splitting than the usual nonrelativistic calculations [12], and similarly for the continuum nucleon the wave function obtained from this equation gives an improved description of nucleon-nucleus elastic scattering data 111 .

\section{Effective Pauli Reduction}

Pauli reduction of the relativistic amplitude for the knock-out contribution to $(\gamma, p)$ reactions was described briefly in reference [6]; we provide more details and discussion here. In the effective Pauli reduction scheme the relativistic distorted wave S matrix is rewritten in terms of Schrödinger-like wave functions [13] resulting in an effective interaction Hamiltonian which may be expanded in powers of $\frac{1}{E+M}$. The various orders can then be related, in the proper limit, to the nonrelativistic form of the amplitude. As we show below there are, however, important differences with the usual nonrelativistic amplitude. The qualitative features of a similar two component reduction of the Dirac wave function for several generic vertices, in the presence of the nuclear interactions, has been discussed by Cooper et al. [14]. The emphasize in our present discussion is to present a more quantitative analysis for the case of $(\gamma, p)$ reactions.

\section{$2.1 \quad$ Formalism}

Beginning with the amplitude of equation (11), we write the integral in terms of Schrödinger-like wave functions using equations (9) and (10) relating the 
Dirac spinors to the Schrödinger-like wave functions. This allows us to write

$$
\begin{aligned}
S_{f i}= & \frac{-i}{(2 \pi)^{3}}\left[\frac{1}{2 \omega}\right]^{1 / 2}\left[\frac{M}{E_{C}}\right]^{1 / 2} \\
& \times \sum_{J_{B} M_{B}}\left(J_{f}, J_{B} ; M_{f}, M_{B} \mid J_{i}, M_{i}\right)\left[\mathcal{S}_{J_{i} J_{f}}\left(J_{B}\right)\right]^{1 / 2} \\
& \times \int \Psi_{\mathrm{Sch}, \mathrm{S}_{f}}^{\dagger(-)}(x) H_{e m}^{e f f}(x) \Psi_{\mathrm{Sch}, J_{B} M_{B}}(x) d^{4} x,
\end{aligned}
$$

where the effective interaction Hamiltonian $H_{I}^{e f f}$ is

$$
\begin{aligned}
H_{I}^{e f f}=D_{C}^{1 / 2}(r) & {\left[1 \frac{\boldsymbol{\sigma} \cdot \boldsymbol{p}}{M+E_{C}+S_{C}(r)-V_{C}(r)}\right] \gamma^{0} H_{e m}(x) } \\
& \times\left[\frac{1}{M+E_{B}+S_{B}(r)-V_{B}(r)}\right] D_{B}^{1 / 2}(r) .
\end{aligned}
$$

This can be expanded in powers of $\frac{1}{E+M}$ and written in the form:

$$
H_{I}^{e f f}=H_{I}^{(1)}+H_{I}^{(2)}+\cdots .
$$

The first and second order contributions are given by

$$
\begin{aligned}
H_{I}^{(1)}= & -\frac{e \kappa}{2 M} \boldsymbol{\sigma} \cdot \boldsymbol{\nabla} \times[\mathbf{A}]-e\left(\frac{1}{M+E_{C}}+\frac{1}{M+E_{B}}\right) \mathbf{A} \cdot \mathbf{p} \\
& +i e\left(\frac{1}{M+E_{C}}-\frac{1}{M+E_{B}}\right) \boldsymbol{\sigma} \cdot \mathbf{A} \times \mathbf{p}-e \frac{\boldsymbol{\sigma} \cdot \boldsymbol{\nabla} \times[\mathbf{A}]}{M+E_{C}} \\
H_{I}^{(2)}= & -\frac{e}{2}\left[\frac{\kappa}{2 M} \boldsymbol{\sigma} \cdot \boldsymbol{\nabla} \times[\mathbf{A}]+\frac{\boldsymbol{\sigma} \cdot \mathbf{A} \boldsymbol{\sigma} \cdot \mathbf{p}}{M+E_{B}}\right] Q_{B}(r) \\
& +e\left[Q_{B}(r)-\kappa \frac{1}{2 M} \omega\right] \boldsymbol{\sigma} \cdot \mathbf{A} \frac{\boldsymbol{\sigma} \cdot \mathbf{p}}{M+E_{B}} \\
& -\frac{e}{2} Q_{C}(r)\left[\frac{\kappa}{2 M} \boldsymbol{\sigma} \cdot \boldsymbol{\nabla} \times[\mathbf{A}]+\frac{\boldsymbol{\sigma} \cdot \mathbf{A} \boldsymbol{\sigma} \cdot \mathbf{p}}{M+E_{B}}\right. \\
& \left.\quad+\frac{\boldsymbol{\sigma} \cdot \mathbf{p} \boldsymbol{\sigma} \cdot \mathbf{A}}{M+E_{C}}\right] \\
& +e \frac{\boldsymbol{\sigma} \cdot \mathbf{p}}{M+E_{C}}\left\{Q_{C}(r)-\frac{1}{2} Q_{B}(r)+\frac{\kappa}{2 M} \omega\right\} \boldsymbol{\sigma} \cdot \mathbf{A},
\end{aligned}
$$


where we have written

$$
Q_{X}(r)=\frac{S_{X}(r)-V_{X}(r)}{E+M}
$$

$\mathbf{O}[f] \cdots$ in the interaction Hamiltonians of equation (19) means that operator $\mathrm{O}$ acts only on function $f$. The appearance of the Dirac potentials through Q's in the interaction Hamiltonians (19) delineates the modification of the effective photon interaction due to the presence of the nuclear medium. We will investigate the significance of this medium effect in the following section.

Using the Hamiltonian (19) in equation (16) along with the nonrelativistic wave functions, we can cast the amplitude in a nonrelativistic form as follows

$$
\begin{aligned}
S_{f i}^{(i)}=\frac{-i}{(2 \pi)^{3}}[ & \left.\frac{1}{2 \omega}\right]^{1 / 2} \sum_{J_{B} M_{B}}\left(J_{f}, J_{B} ; M_{f}, M_{B} \mid J_{i}, M_{i}\right)\left[\mathcal{S}_{J_{i} J_{f}}\left(J_{B}\right)\right]^{1 / 2} \\
& \times \int \Psi_{\mathrm{Sch}, \mathrm{S}_{f}}^{\dagger(-)}(x) H_{I}^{(i)}(x) \Psi_{\mathrm{Sch}, J_{B} M_{B}}(x) d^{4} x
\end{aligned}
$$

where $(i)$ refers to the highest order of the inverse of the nucleon mass in the interaction Hamiltonian used in the nonrelativistic amplitude. The amplitude (21), with $H_{I}^{i}=H_{I}^{(1)}$ in the limit $E_{B}, E_{C} \rightarrow M$ is equivalent to the usual nonrelativistic transition amplitude [17] except that the Schrödingerlike wave functions are used instead of the usual nonrelativistic Schrödinger wave functions. The amplitude obtained this way will be referred to here as the first order nonrelativistic amplitude.

The Schrödinger-like wave functions describing the bound nucleon can be written as

$$
\Psi_{\mathrm{Sch}, \mathrm{B}}(x)=e^{-i E_{B} t} f_{L_{B}}(r) \mathcal{Y}_{L_{B} 1 / 2 J_{B}}^{M_{B}}(\Omega),
$$

while for the continuum nucleon we write

$$
\begin{aligned}
\Psi_{\mathrm{Sch}, \mathrm{C}}^{\dagger}(x)=4 \pi e^{i E_{C} t} & \sum_{L M J} i^{-L} Y_{L}^{M-S_{f}}\left(\hat{k}_{f}\right) \\
& \times\left(L, 1 / 2 ; M-S_{f}, S_{f} \mid J, M\right) f_{L J}(r) \mathcal{Y}_{L 1 / 2 J}^{M \dagger}(\Omega),
\end{aligned}
$$

where

$$
\mathcal{Y}_{L 1 / 2 J}^{M}(\Omega)=\sum_{\mu}(L, 1 / 2 ; M-\mu, \mu \mid J, M) Y_{L}^{M-\mu}(\Omega) \chi_{1 / 2}^{\mu} .
$$


It should be emphasized here that the wave functions introduced in equations (22) and (23) have nonrelativistic normalization, i.e the factor $\sqrt{\frac{E+M}{2 M}}$ which comes from the Dirac spinor describing the outgoing nucleon has been set equal to one (thus a bound state wave function is normalized to one and the plane wave limit of the nucleon wave function is of the form $\Psi_{\mathrm{Sch}, \mathrm{S}_{f}}(x)=$ $\left.e^{-i k \cdot x} \chi_{1 / 2}^{S_{f}}\right)$. In addition the factor $\sqrt{\frac{M}{E}}$ which comes from the Dirac field expansion is set equal to one in the cross section.

Using equations (3), (22) and (23) then after evaluating the angular integration the first order amplitude can be written as

$$
\begin{aligned}
S_{f i}^{(1)}= & \frac{-i e}{\pi}\left[\frac{1}{2 \omega}\right]^{1 / 2} \sum_{J_{B} M_{B}}\left(J_{f}, J_{B} ; M_{f}, M_{B} \mid J_{i}, M_{i}\right)\left[\mathcal{S}_{J_{i} J_{f}}\left(J_{B}\right)\right]^{1 / 2} \\
\times & \delta\left(E_{C}-E_{B}-\omega\right) \sum_{l L J \mu}(-i)^{l+L}(2 l+1) \\
\times & \left\{\left(L, 1 / 2 ; M_{B}+2 \mu-S_{f}, S_{f} \mid J, M_{B}+2 \mu\right) Y_{L}^{M_{B}+2 \mu-S_{f}}\left(\hat{k}_{f}\right)\right. \\
\times & {\left[\left(\frac{\kappa \omega}{2 M}+\frac{\omega}{M+E_{C}}\right) I_{l, L, J, L_{B}} C_{l, L, J, L_{B}}^{\mu}\right.} \\
& \quad(2 \mu \cos \xi-i \sin \xi)\left(\frac{1}{M+E_{C}}-\frac{1}{M+E_{B}}\right) \\
+ & \sum_{\nu= \pm 1}\left(L, 1 / 2 ; M_{B}-\nu-S_{f}, S_{f} \mid J, M_{B}-\nu\right) Y_{L}^{M_{B}-\nu-S_{f}}\left(\hat{k}_{f}\right) \\
& \times\left[\left(\frac{1}{M+E_{C}}+\frac{1}{M+E_{B}}\right)+2 \nu \mu\left(\frac{1}{M+E_{C}}-\frac{1}{M+E_{B}}\right)\right] \\
& \left.\times H_{L, J, L_{B}}^{M_{B}, \mu, \nu}\left[P_{L_{B}+1, l, L}^{M_{B},-\mu, \nu}-\mathcal{P}_{L_{B}-1, l, L}^{M_{B},-\mu, \nu}\right] \frac{\nu \cos \xi+i \sin \xi}{\sqrt{2}}\right\},
\end{aligned}
$$

where $I_{l, L, J, L_{B}}, C_{l, L, J, L_{B}}^{\mu}, P_{L_{B}+1, l, L}^{M_{B}, \mu, \nu}, \mathcal{P}_{L_{B}-1, l, L}^{M_{B}, \mu, \nu}, H_{L, J, L_{B}}^{M_{B} \mu, \nu}$, and $\mathcal{H}_{L, J, L_{B}}^{M_{B}, \mu}$ involve radial integrals and Clebsch-Gordan coefficients. These functions are defined in appendix A. The corresponding expression for the amplitude to second order in $\frac{1}{E+M}$ has the same structure as (25) but the contributing terms are more complicated. 


\subsection{Results of the Effective Pauli Reduction}

In the previous subsection we illustrated how the effective Pauli reduction of the relativistic amplitude for the knock-out contribution to $(\gamma, p)$ reactions is performed to get an expansion in powers of $\frac{1}{E+M}$. The successive terms in this expansion can be reduced, in the appropriate limits, to forms that are equivalent to the amplitudes used in nonrelativistic calculations. This allows us to carry out quantitative comparisons between the relativistic and nonrelativistic calculations. The appropriate limits referred to above include i) setting the nucleon total energy equal to its rest mass ii) turning off the nuclear potentials in the second-order interaction Hamiltonian iii) taking proper account of wave function normalizations. This comparison will be carried out for the differential cross section as well as the photon asymmetry [15] at representative energies. We shall compare the following four types of calculations:

a) Full relativistic calculations using the amplitude given by equation (11). In the figures that follow these calculations are represented by solid curves and denoted "Relativistic". For these calculations the relativistic $(\gamma, p)$ code of Lotz has been used 16.

b) First order nonrelativistic calculations. These calculations are obtained using the amplitude $S_{f i}^{(1)}$ of equation (25). Note that in the limit $E_{B}$ and $E_{C} \rightarrow M$, the interaction Hamiltonian takes on a simplified form. These calculations essentially represent the standard nonrelativistic calculations. Comparison of these results with the relativistic calculations gives the essence of the difference between the two approaches. These calculations are denoted "Pauli N.R. (First order)" in Figs. 1 and 2 and are represented by the dotted curves.

c) The third type of calculation represents a nonrelativistic calculation carried out to second order in the inverse nucleon mass. This calculation then includes the interaction Hamiltonian $H_{I}^{(2)}$, but with the nuclear vector and scalar potential set equal to zero in the interaction Hamiltonian. This interaction will then relate to the limit in which the photon is interacting with a free nucleon. Our intention here is to clarify how much improvement in the nonrelativistic calculations can be obtained by including second order effects. We shall show that the effects are not substantial. 
These results are shown by the dot-dashed curves in Figs. 1 and 2 and are labelled "Pauli N.R. (First + Second)". We shall refer to these calculations in the text as "medium-uncorrected" calculations to signify the fact that they pertain to the limit in which the nuclear potentials are set to zero.

d) The fourth type of calculation represents a nonrelativistic calculation using the full expression for $H_{I}^{(2)}$, i.e. with the effect of the nuclear medium (through the presence of the potentials) taken into account. These calculations are shown by the dashed curves in Figs. 1 and 2 and are labelled "Pauli N.R. (First + Full Second)". They will be referred to as "medium-corrected" nonrelativistic calculations in the following text. The essence of the present comparison is to show the significance of these medium effects.

Since our aim in this paper is to compare the two theoretical models, namely relativistic and nonrelativistic, for the knock-out contribution to $(\gamma, p)$ reactions, we do not compare the resulting observables with data. We refer the reader to the work of Lotz and Sherif [1] for comparison of the results of the relativistic model with data.

The bound state wave functions used in the calculations reported in this section are generated using the Dirac-Hartree potentials of Horowitz and Serot [12]. In all the calculated cross sections given in this paper the spectroscopic factor takes its maximum allowable value of $\left(2 J_{B}+1\right)$ for both the relativistic and nonrelativistic calculations.

Figure 1 shows the calculated observables for the ${ }^{16} O(\gamma, p){ }^{15} N$ reaction with a photon energy of $E_{\gamma}=100 \mathrm{MeV}$. The residual nucleus is in a $1 p_{\frac{1}{2}}$ single hole state. The final state optical potential is taken from reference [16]. The cross section curves of Fig. 1(a) show large differences between the first order nonrelativistic (dotted curve) and the relativistic calculations (solid curve). At forward angles the nonrelativistic calculations are almost an order of magnitude larger than the relativistic calculations, while at backward angles the nonrelativistic calculations are roughly two orders of magnitude smaller than the relativistic calculations. Medium-uncorrected second order calculations improve the nonrelativistic calculations slightly only at forward angles (dot-dashed curve). On the other hand the medium-corrected second order calculations produce large changes in the nonrelativistic calculations. 
Note the large change in the magnitude for both small and large scattering angles (dashed curve) which brings the nonrelativistic calculations into close agreement with the results of the relativistic model.

Calculations of photon asymmetry shown in Fig. 1(b) also exhibit noticeable differences between the first order nonrelativistic and relativistic calculations. These two calculations have different shapes and magnitudes especially for scattering angles greater than $40^{\circ}$. Medium-uncorrected second order calculations modify the nonrelativistic calculations slightly in magnitude while the medium-corrected second order calculations result in noticeable changes in the shape and magnitude of the nonrelativistic calculations. These changes are such that at forward angles the nonrelativistic calculations now overlap the relativistic calculations and are much closer in shape and magnitude at backward angles.

Figure 2 shows similar comparisons for the reaction at a higher incident photon energy, $E_{\gamma}=312 \mathrm{MeV}$. The final state global optical potentials are taken from Cooper et al. 11]. Figure 2(a) shows that the cross section obtained from the first order nonrelativistic calculations has both different shape and magnitude from the results of the relativistic calculations. The nonrelativistic calculations lie above the relativistic calculations for angles smaller than $40^{\circ}$, whereas for other scattering angles the nonrelativistic calculations lie below the relativistic calculations by as much as an order of magnitude. Medium-uncorrected second order calculations lie closer to the relativistic calculations only at forward angles. Medium-corrected second order calculations, on the other hand, are much closer to the results of the relativistic calculations.

Calculations of the photon asymmetries of Fig. 2(b) show that the first order nonrelativistic results differ from the relativistic calculations in both shape and magnitude. Medium-uncorrected second order calculations improve the nonrelativistic calculations slightly at forward angles but the overall shape stays the same as that of the first order. Medium-corrected second order calculations modify the shape and magnitude of the nonrelativistic calculations at all scattering angles. It might not be clear visually that these changes bring the nonrelativistic calculations into noticeably better agreement with the relativistic ones, however a chi-squared comparison does indeed show that the medium-corrected second order calculations are closer to the relativistic calculations than medium-uncorrected second order calculations. The important point here is that there are large differences in the 
calculations when medium corrections are taken into account.

From these examples one can see that the first order nonrelativistic calculations are different from relativistic calculations. Attempts to improve the situation through the inclusion of the second order terms in which the presence of the nuclear potentials is neglected (as would normally be done in typical nonrelativistic calculations) are bound to fail in bringing the results close to the relativistic calculations. We have seen that medium-corrected second order calculations (i.e. those that include the effects of the nuclear potentials on the interaction Hamiltonian) are much closer. We remind the reader that these potentials are absent in interaction terms of the usual nonrelativistic calculation even when higher order relativistic corrections are included [17]. This medium modification is the important ingredient that is missing in ordinary nonrelativistic calculations. It is this medium modification that is responsible for many of the differences between the two types of calculations. In the following section we show that the same conclusion can be reached through a procedure based on the Foldy Wouthuysen transformation.

\section{Foldy-Wouthuysen Transformation}

Following McVoy and Van Hove [5] many authors construct a nonrelativistic model of photons interacting with nuclei by performing a FW transformation on the relativistic Hamiltonian which involves the electromagnetic interaction with a free nucleon. The resulting nonrelativistic interaction Hamiltonian is then sandwiched between Schrödinger wave functions describing the initial and final nucleons [17]. To investigate the effect of the nuclear medium on the FW reduction of the relativistic amplitude of the knock-out contribution to $(\gamma, p)$ reactions, we perform a $\mathrm{FW}$ transformation on the relativistic Hamiltonian of a photon interacting with a nucleon in the presence of strong scalar and vector potentials.

In the preceding discussion of the Pauli reduction scheme, our starting point was the distorted wave amplitude of equation (11). A feature of this amplitude for practical calculation is the use of complex vector and scalar potentials to describe the interaction of the outgoing nucleon with the residual nucleus. The unitary requirement for the FW transformation makes it unacceptable to work with this type of amplitude (note that in the usual DWBA amplitude the initial and final states of the nucleon are described by 
different Hamiltonians). We must therefore work with a model amplitude in which the bound and continuum state potentials are the same and real. It is known that such model amplitudes are inferior in their description of the data in comparison to the distorted wave amplitudes. It must be noted however that the purpose of the present investigation is not aimed at fitting data; rather we are interested in features that differentiate between the relativistic and nonrelativistic calculations. For this purpose the restricted model amplitude used here is quite appropriate.

\subsection{Spin $\frac{1}{2}$ Particle Interacting with Strong and Elec- tromagnetic Potentials}

We first summarize the FW transformation for the case in which a Dirac particle interacts with a general field following the procedure as given in the reference [9]. The results will then be applied to the case in which a nucleon interacts with an electromagnetic field while under the influence of the strong potentials. The Dirac equation is written in the general form

$$
i \partial_{t} \psi(x)=H \psi(x),
$$

where the relativistic Hamiltonian $H$ can be written in terms of even and odd operators as

$$
H=\beta M+\mathcal{E}+\mathcal{O} .
$$

The odd operator $\mathcal{O}$ connects the upper component of the Dirac spinor to the lower component while the even operator $\mathcal{E}$ can only connect either upper or lower components. Now we perform the FW transformation on the relativistic Hamiltonian (27) following reference [9]. After three successive transformations we find.

$$
\begin{aligned}
H^{\prime}=\beta M & +\mathcal{E}+\frac{\beta}{2 M} \mathcal{O}^{2}-\frac{i}{8 M^{2}}[\mathcal{O}, \dot{\mathcal{O}}] \\
& -\frac{1}{8 M^{2}}[\mathcal{O},[\mathcal{O}, \mathcal{E}]]-\frac{\beta}{8 M^{3}} \mathcal{O}^{4}
\end{aligned}
$$

(For simplicity the transformed Hamiltonian is denoted $H^{\prime}$, this is in fact the same as the Hamiltonian $H^{\prime \prime \prime}$ in reference [9].) This result applies to any pair 
of even and odd operators. We are interested here in a transformed Hamiltonian containing terms which are even up to second order in the inverse of the nucleon mass so we will eventually drop the last term in equation (28).

The relativistic Hamiltonian for a proton interacting with an external electromagnetic field while under the influence of the strong vector and scalar nuclear potentials can be written as

$$
H=\boldsymbol{\alpha} \cdot \boldsymbol{\pi}+e \Phi+\beta[M+S(r)]+V(r)+\frac{e \kappa}{2 M} \beta \sigma^{\mu \nu} F_{\mu \nu},
$$

where $\boldsymbol{\pi}=\mathbf{p}-e \mathbf{A}$. The above Hamiltonian can be written in terms of the electric and magnetic fields $\mathbf{E}$ and $\mathbf{B}$ as

$$
H=\boldsymbol{\alpha} \cdot \boldsymbol{\pi}+\beta[M+S(r)]+V(r)+\frac{i \kappa e}{2 M}(\boldsymbol{\gamma} \cdot \mathbf{E}+i \boldsymbol{\Sigma} \cdot \mathbf{B}),
$$

where we have defined

$$
\boldsymbol{\Sigma}=\beta \boldsymbol{\sigma} .
$$

We rewrite the Hamiltonian (30) in terms of even and odd operators as in equation (27). In this case the even and odd operators are

$$
\mathcal{E}=\beta S(r)+V(r)-\frac{\kappa e}{2 M} \boldsymbol{\Sigma} \cdot \mathbf{B},
$$

and

$$
\mathcal{O}=\boldsymbol{\alpha} \cdot \boldsymbol{\pi}+\frac{i e \kappa}{2 M} \boldsymbol{\gamma} \cdot \boldsymbol{E}
$$

These even and odd operators are used in equation (28) in order to get the FW transformed Hamiltonian for a nucleon interacting with electromagnetic and strong potentials. We remind the reader that the quality of the FW transformation depends on the assumption that the potential depths are small compared with the nucleon mass; $V, S \ll M$. This is not the case for the Dirac potentials, and so we are by no means guaranteed that the FW transformation will yield a convergent series. We will return to this point below.

The resulting Hamiltonian to second order in the inverse of the nucleon mass is a $4 \times 4$ block diagonal matrix

$$
H^{\prime}=\left[\begin{array}{cc}
H_{11}^{\prime} & 0 \\
0 & H_{22}^{\prime}
\end{array}\right]
$$


The upper left element of this matrix (i.e $H_{11}^{\prime}$ ) corresponds to the transformed Hamiltonian for positive energy solutions of the Dirac wave function. For the nonrelativistic limit we will use this part of the Hamiltonian which we write as

$$
H_{11}^{\prime}=H_{0}+H_{I}
$$

where $H_{0}$ involves strong potentials whereas $H_{I}$ carries both strong and electromagnetic interactions: we will treat the latter as a perturbation on the former. These Hamiltonians are evaluated explicitly using the Coulomb gauge. $H_{0}$ can be written to second order in $1 / M$ as

$$
\begin{aligned}
H_{0}= & \frac{\mathbf{p}^{2}}{2 M}+M+S(r)+V(r) \\
& -\frac{1}{4 M^{2} r}\left[S^{\prime}(r)-V^{\prime}(r)\right] \boldsymbol{\sigma} \cdot \mathbf{L} \\
& -\frac{1}{2 M^{2}}\left\{S(r) \mathbf{p}^{2}+\mathbf{p}[S(r)] \cdot \mathbf{p}+\frac{\mathbf{p}^{2}[V(r)+S(r)]}{4}\right\} .
\end{aligned}
$$

The interaction Hamiltonian is written in orders of $1 / M$ as

$$
H_{I}=H_{I}^{(1)}+H_{I}^{(2)}+\cdots,
$$

where for the first and second orders we have

$$
\begin{aligned}
H_{I}^{(1)}= & -\frac{e}{M} \mathbf{A} \cdot \mathbf{p}-\frac{e}{2 M}(1+\kappa) \boldsymbol{\sigma} \cdot(\boldsymbol{\nabla} \times \mathbf{A}) \\
H_{I}^{(2)}= & \frac{e \omega}{8 M^{2}}(1+2 \kappa)\{\boldsymbol{\sigma} \cdot \boldsymbol{\nabla} \times[\boldsymbol{A}]-2 i \boldsymbol{\sigma} \cdot \mathbf{A} \times \mathbf{p}\} \\
& +\frac{e}{4 M^{2}}\left\{\begin{array}{c}
2 S(r) \mathbf{A} \cdot \mathbf{p}+2 \mathbf{A} \cdot \mathbf{p} S(r)+2 S(r) \boldsymbol{\sigma} \cdot \boldsymbol{\nabla} \times[\mathbf{A}] \\
+\boldsymbol{\sigma} \cdot \mathbf{A} \times \boldsymbol{\nabla}[V(r)-S(r)] \\
-(V(r)-S(r)) \boldsymbol{\sigma} \cdot \boldsymbol{\nabla} \times[\mathbf{A}]\} .
\end{array}\right.
\end{aligned}
$$

Note that in equation (38) terms of order $e^{2}$ are dropped, and as before $\mathbf{O}[f] \cdots$ means that operator $\mathbf{O}$ operates only on function $f$. 


\subsection{Schrödinger-like Wave Functions}

The wave functions describing either the bound or continuum nucleons are obtained by solving the equation

$$
H_{0} \Psi(\mathbf{r})=E \Psi(\mathbf{r})
$$

where $H_{0}$ is the Hamiltonian (36) containing terms to second order in $1 / M$, and $\mathrm{E}$ is the total energy of the nucleon. Note that $H_{0}$ contains a first derivative term which can be eliminated using the transformation

$$
\Psi(\mathbf{r})=D_{\mathrm{FW}}^{-\frac{1}{2}}(r) \Psi_{\mathrm{Sch}}(\mathbf{r})
$$

where $D_{\mathrm{FW}}=1-\frac{S(r)}{M}$. With this choice of $D_{\mathrm{FW}}$, the two functions $\Psi(\mathbf{r})$ and $\Psi_{\text {Sch }}(\mathbf{r})$ have the same asymptotic form. After this transformation the wave equation (39) takes the form of the Schrödinger-like equation (12). The central and spin-orbit potentials in the present case are

$$
\begin{aligned}
U_{\text {cent }}(r)=\quad \frac{1}{D_{\mathrm{FW}}}\{S(r)+ & V(r)+\frac{1}{4 M^{2} r}\left[S^{\prime}(r)+V^{\prime}(r)\right] \\
& \left.\quad+\frac{1}{8 M^{2}}\left[S^{\prime \prime}(r)+V^{\prime \prime}(r)\right]\right\} \\
& +(E-M)\left\{1-\frac{1}{D_{\mathrm{FW}}(r)}\right\} \\
& +\frac{1}{2 M r} \frac{D_{\mathrm{FW}}^{\prime}(r)}{D_{\mathrm{FW}}(r)}+\frac{1}{4 M} \frac{D_{\mathrm{FW}}^{\prime \prime}(r)}{D_{\mathrm{FW}}(r)}-\frac{1}{8 M}\left(\frac{D_{\mathrm{FW}}^{\prime}(r)}{D_{\mathrm{FW}}(r)}\right)^{2}, \\
U_{\mathrm{so}}(r)= & \frac{1}{D_{\mathrm{FW}}(r)}\left\{-\frac{1}{4 M^{2} r}\left[S^{\prime}(r)-V^{\prime}(r)\right]\right\} .
\end{aligned}
$$

The nonrelativistic amplitude for the knock-out contribution to the $(\gamma, p)$ reaction at the desired order of $1 / M$ takes the same form as given in equation (21). Note however that the second order amplitude obtained through the FW scheme involves wave functions which are solutions of equation (39). As we have seen above, this equation does not have the form of the usual Schrödinger wave equation because it contains a first order derivative of the wave function. To be consistent with the usual nonrelativistic formalism we rewrite the second order amplitude in terms of the Schrödinger-like wave 
functions $\Psi_{\mathrm{Sch}}(\mathbf{r})$ introduced in equation (40). This requires that the interaction Hamiltonian be modified to

$$
H_{I}^{\prime}=D_{\mathrm{FW}}^{-\frac{1}{2}} H_{I} D_{\mathrm{FW}}^{-\frac{1}{2}} \text {. }
$$

With this modification the first order terms in the interaction Hamiltonian will remain the same as $H_{I}^{(1)}$ in (38), while the second order terms in the interaction Hamiltonian become

$$
\begin{gathered}
H_{I}^{(2)^{\prime}=} \frac{e \omega}{8 M^{2}}(1+2 \kappa)\{\boldsymbol{\sigma} \cdot \boldsymbol{\nabla} \times[\boldsymbol{A}]-2 \boldsymbol{\sigma} \cdot \mathbf{A} \times \boldsymbol{\nabla}\} \\
-\frac{e}{4 M^{2}}\{2 \kappa S(r) \boldsymbol{\sigma} \cdot \boldsymbol{\nabla} \times[\mathbf{A}]+\boldsymbol{\sigma} \cdot \mathbf{A} \times \boldsymbol{\nabla}[V(r)-S(r)] \\
-(V(r)-S(r)) \boldsymbol{\sigma} \cdot \boldsymbol{\nabla} \times[\mathbf{A}]\}
\end{gathered}
$$

The calculations of the amplitudes proceed in the same manner discussed in section 2. In particular the amplitude has the same form as that of equation (21), but now the interaction Hamiltonian is that of equation (43) while the wave functions are solutions of a Schrödinger-like equation using the central and spin-orbit potentials of equation (41). Note that at first order the FW formalism does not produce an acceptable nucleon wave function since there is only a central potential and no spin-orbit potential, see equation (41). The spin-orbit potential is borrowed from the second order and used with the first order terms to get nucleon wave functions for use in the first order which at least have a reasonable form.

\subsection{Differences Between the FW and Pauli Reduction Schemes}

The Pauli reduction scheme begins with the relativistic distorted wave amplitude in which the initial and final nucleons are described by Dirac wave functions. The Dirac wave functions are solutions of the Dirac wave equation (6) with different strong potentials for bound and continuum nucleons.

The amplitude resulting from the Pauli reduction procedure has bound and continuum nucleons described by nonrelativistic wave functions. These wave functions are solutions of the Schrödinger-like equation (12) with central 
and spin-orbit potentials which in turn, are functions of the Dirac potentials $S(r)$ and $V(r)$.

In the FW scheme the initial bound and final continuum nucleons are described by the same Hamiltonian. Due to the unrealistic potentials used to describe the continuum nucleon, the FW calculations provide a toy model which will shed more light on the differences between the relativistic and nonrelativistic calculations. We should stress that unlike the Pauli reduction in which the wave functions describing nucleons are the same in all the calculations of different orders, in the FW transformation the nucleon wave functions are changed in each order of calculation due to the contribution of different terms to the wave equation. Differences between these two reduction formalisms in the free nucleon limit has been studied by Fearing et al. [18]. They find that for the interaction of a real photon with a free particle at first order in the coupling constant the FW and Pauli reductions produce the same nonrelativistic interaction Hamiltonian.

\subsubsection{Differences between the Pauli and FW Hamiltonians}

In order to make instructive comparisons between the Pauli and FW Hamiltonians we need to simplify the Hamiltonians obtained through the Pauli reduction scheme of section 2. First the potentials describing the initial and final nucleons are taken to be the same; in particular they will be the real Hartree potentials used to describe the bound state in the relativistic calculations. In addition, the energies of the bound and continuum nucleons are set equal to the nucleon mass in the Pauli interaction Hamiltonians. In this limit the first order terms in the FW and Pauli interaction Hamiltonians are exactly the same. Differences will appear in terms of order $\frac{1}{M^{2}}$ and higher. The difference between the interaction terms of the two schemes up to second order, with the above modifications of $H_{I}^{\text {Pauli }}$, is

$$
\begin{aligned}
H_{I}^{\mathrm{FW}}-H_{I}^{\mathrm{Pauli}}= & \frac{e \omega}{8 M^{2}}\{\boldsymbol{\sigma} \cdot(\boldsymbol{\nabla} \times \boldsymbol{A})-2 \boldsymbol{\sigma} \cdot(\mathbf{A} \times \boldsymbol{\nabla})\} \\
& -\frac{e \kappa}{4 M^{2}}\{[S(r)+V(r)] \boldsymbol{\sigma} \cdot(\boldsymbol{\nabla} \times \mathbf{A})\} .
\end{aligned}
$$

In the special case describing the interaction of a photon with a free nucleon, i.e in the limit when the strong potentials $S(r)$ and $V(r)$ are set equal to zero, equation (44) agrees with the results of Fearing et al. [18]. Detailed calculations show that the first term on the right hand side of equation (44) 
is very small for photon energies less than a few hundred $\mathrm{MeV}$, but becomes more important at higher energies. The second term involves the sum of scalar and vector potentials. At the origin the sum of these potentials is about $-100 \mathrm{MeV}$. Note that the square of the nucleon mass $(\sim 1000 \mathrm{MeV})$ appears in the denominator of this term, so it has a very small coefficient, with the result that the second term on the right hand side of the above equation also makes only a small contribution to the transition amplitude. Thus the differences between the FW and Pauli interaction Hamiltonians appear to be small if we restrict ourselves to second order in the inverse nucleon mass.

The wave functions describing nucleons in the Pauli scheme are the same for all orders of calculations while in the FW scheme the wave functions change at every order. The FW central potential at second order is similar in shape and energy dependence to the central potential obtained via the Pauli formalism. A very interesting point to note is that there is no spinorbit potential at first order in the FW formalism: it appears at second order with a shape similar to that obtained in the Pauli scheme, but its magnitude is roughly a factor of two smaller than the spin-orbit potential arising in the Pauli scheme.

\subsection{Results of the FW Reduction}

In this section we compare the theoretical results of the relativistic approach with those of the nonrelativistic amplitudes obtained through FW reduction. The results are shown for three different incident photon energies. As in the Pauli discussion of section 2.2 we present three different types of nonrelativistic calculations, namely: first order, medium-uncorrected second order and medium-corrected second order. Recall that in the first order nonrelativistic calculations the wave functions are solutions of equation (39), ignoring all the second order terms in $H_{0}$ (equation (36)) except for the spin-orbit potential. In the second order calculations the wave functions are obtained from equation (39) with all the first and second order termsin $H_{0}$ included. The Hartree potentials used in all calculations (relativistic and nonrelativistic) are from reference [12]. The graphs discussed in this section are labeled as in the Pauli discussion of section 2.2 except for an obvious change of notation.

Figure 3 shows the calculated observables for the ${ }^{16} O(\gamma, p){ }^{15} \mathrm{~N}$ reaction for a photon of energy $E_{\gamma}=100 \mathrm{MeV}$. Figure $3(\mathrm{a})$ shows the cross 
sections. At small angles the first order nonrelativistic calculations (dotted curve) are about an order of magnitude lower than the relativistic calculations (solid curve), while for large angles the first order calculations lie above the relativistic calculations. Medium-uncorrected second order calculations (dot-dashed curve) show substantial increase in the the magnitude of the cross sections at small scattering angles as well as some change in the shape of the resulting curve. Medium-corrected second order calculations (dashed curve) produce a noticeable change in the cross sections at backward angles.

The photon asymmetry calculations of Fig. 3(b) also show noticeable differences between the first order nonrelativistic and relativistic calculations at backward angles. Medium-uncorrected second order calculations produce a change in the magnitude and the shape of the asymmetry for scattering angles greater than $80^{\circ}$. Medium-corrected second order calculations produce a shift towards larger angles resulting in a qualitatively similar shape to that of the relativistic calculations.

Figure 4 shows the observables for the same reaction as Fig. 3 but the photon energy in this case is $E_{\gamma}=196 \mathrm{MeV}$. The cross section results are shown in Fig. 4(a), where we note that the first order nonrelativistic calculations are generally lower than the relativistic calculations by one to two orders of magnitude. They also fail to reproduce the dip near mid-angles. Second order calculations lead to a drastic change in the cross section with large differences due to medium corrections at both forward and backward angles. Medium-corrected second order calculations are in noticeably closer agreement with relativistic calculations compared to the medium-uncorrected ones.

Similar features are observed for the photon asymmetry calculations (Fig. $4(\mathrm{~b})$ ). Here again we notice large differences between the relativistic and first-order nonrelativistic calculations. Large differences also exist between medium-corrected and medium-uncorrected calculations. The level of agreement between the second order medium-corrected calculations and the relativistic calculations is not the same as observed in the case of cross sections.

The calculations shown in Fig. 5 for a photon energy of $E_{\gamma}=312 \mathrm{MeV}$ show essentially the same qualitative features.

One characteristic that emerges from the above discussion is that the full second order calculations (medium-corrected calculations) in the FW scheme are not as close to the relativistic results as in the Pauli reduction case at the same photon energy. This brings out an essential difference between the Pauli 
and FW calculations: The wave functions in the Pauli formalism remain the same while different orders of the amplitude result solely from the expansion of the interaction Hamiltonian. The FW calculations, on the other hand, involve an expansion affecting both the wave functions and the interaction Hamiltonian simultaneously. This difference is at the root of the different convergence properties of the two approaches. We find that in most cases, by second order the Pauli expansion is quite close to the fully relativistic

calculations, provided medium corrections are taken into account. In the FW scheme the level of agreement at the corresponding order is inferior, indicating that the convergence in this scheme is much slower than in the Pauli case.

\section{Conclusions}

We have described two different nonrelativistic reduction schemes of the relativistic amplitude describing the knock-out contribution to $(\gamma, p)$ reactions. These reductions allow us to carry out controlled comparisons between the relativistic and nonrelativistic calculations of the reaction observables. In the Pauli formalism the relativistic $\mathrm{S}$ matrix is written in terms of nonrelativistic two-component wave functions and an effective interaction Hamiltonian. The effective Hamiltonian is expanded in powers of $1 /(E+M)$, where $M$ is the nucleon mass and $E$ is its total energy. In the limit $E \rightarrow M$, the first order interaction terms are exactly the same as those appearing in the usual nonrelativistic amplitude. The nonrelativistic wave functions in this scheme are solutions of the Schrödinger-like wave equation (12). Detailed comparisons between the relativistic and first-order nonrelativistic predictions for the differential cross sections and photon asymmetries show large differences between the two types of calculations. The inclusion of terms to second order in $1 / M$ in the interaction Hamiltonian, where medium corrections effected by the nuclear potentials are left out, does not lead to any substantial improvement in the agreement between the relativistic and nonrelativistic calculations. On the other hand the expansion scheme shows explicit dependence in the second order terms on the nuclear potentials. When these medium corrections are taken into account the nonrelativistic calculations converge close to the relativistic results. This indicates that the essential difference between the relativistic and traditional nonrelativistic amplitudes, 
is the absence in the latter of the medium modification of the interaction Hamiltonian as a consequence of the presence of the strong vector and scalar potentials.

These conclusions are further supported through an analysis based on the Foldy-Wouthuysen transformation of the relativistic Hamiltonian describing a photon interacting with a nucleon embedded in the nuclear medium. The nonrelativistic wave functions for the bound and continuum nucleons are solutions of the wave equation obtained as a result of the transformation. The scheme leads to a nonrelativistic amplitude calculated to the desired order in $1 / M$. We use these amplitudes to carry out comparisons between relativistic and nonrelativistic calculations in the manner described above for the Pauli scheme. We find that the medium modifications in the second order calculations are important and their inclusion leads in general to better agreement with the relativistic calculations. However the convergence is not as efficient at this order as in the Pauli case. The reasons for this can be understood in terms of the formal differences between the structure of the nonrelativistic amplitude obtained using this transformation as compared to the Pauli reduction case. The wave functions obtained through the FW reduction are different at each order in $1 / M$, in contrast to the Pauli wave functions which are unchanged for all orders (recall that in the Pauli reduction only the interaction Hamiltonian is expanded).

The basic result of the present work is that standard nonrelativistic calculations of the knock-out amplitude do not properly take into account the strong medium modifications of the interaction Hamiltonian. We have clarified this point through a comparison based on nonrelativistic reduction of the

relativistic amplitude using both the Pauli and Foldy-Wouthuysen reduction schemes.

\section{References}

[1] G.M. Lotz and H.S. Sherif, Phys. Lett. B210 (1988) 45; and Nucl. Phys. A537 (1992) 285.

[2] J.P. McDermott, E. Rost, J.R. Shepard and C.Y. Cheung, Phys. Rev. Lett. 61 (1988) 814. 
[3] R.S. Turley, E.R. Kinney, J.L. Matthews, W.W. Sapp, E.J. Scheidker, R.A. Schumacher, S.A. Wood, G.S. Adams, and R.O. Owens, Phys. Lett. 157B (1985) 19.

[4] L.L. Foldy and S.A. Wouthuysen, Phys. Rev. 78 (1950) 29.

[5] K.W. Mcvoy and L.Van Hove, Phys. Rev. 125 (1962) 1034.

[6] M. Hedayati-Poor and H.S. Sherif, Phys. Lett. B326 (1994) 9.

[7] M. Hedayati-Poor, J.I. Johansson and H.S. Sherif, Phys. Rev. C 51 (1995) 2044.

[8] H.W.L. Naus and J.H. Koch, Phys. Rev. C 36 (1987) 2459.

[9] J.D. Bjorken and S.D. Drell, Relativistic Quantum Mechanics, (McGraw-Hill Book Company 1964).

[10] B.D. Serot and J.D. Walecka, Advances in Nuclear Physics (J.W. Negele and E. Vogt,eds.) Vol.16, Plenum Press, New York (1986)

[11] E.D. Cooper, S. Hama, B.C. Clark and R.L. Mercer, Phys. Rev. C 47 (1993) 297. (1981) 91.

[12] C.J. Horowitz and B.D. Serot, Nucl. Phys. A368 (1986) 503.

[13] B.C. Clark, S. Hama and R.L. Mercer, AIP conf. proc. N. 97, ed: H.O. Mayer (1982) 260; J. Raynal, Aust. J. Phys. 43 (1990) 9; G.Q. Li, J. Phys. G19 (1993) 1841.

[14] E.D. Cooper, A.O. Gattone and M.H. Macfarlane, Phys. Lett. B130 (1983) 359.

[15] V.B. Ganenko, V.A. Gushchin, Yu. V. Zhebrovskii, L. Ya. Kolesnikov, A.L. Rubashkin and P.V. Sorokin, JETP Lett. 47 (1988) 519; G.S. Blanpied et al., Phys. Rev. Lett. 67 (1991) 1206.

[16] Gerhard Martin Lotz, Ph.D. thesis, University Of Alberta, 1989.

[17] S. Boffi, C. Giusti and F.D. Pacati, Nucl. Phys. A359

[18] H.W. Fearing, G.I. Poulis and S. Scherer, Nuc. Phys. A570, (1994) 657. 


\section{Appendix A}

In this appendix we give the explicit form of the six functions involving Clebsch-Gordan coefficients and radial integrals which are introduced in the amplitude of equation (25).

$$
\begin{aligned}
& I_{l, L, J, L_{B}}=\left(L_{B}, l ; 0,0 \mid L, 0\right) \int r^{2} d r f_{B}(r) j_{l}\left(k_{\gamma} r\right) f_{L J}(r), \\
& P_{L_{B}+1, l, L}^{M_{B}, \mu, \nu}=\sqrt{\frac{L_{B}+1}{2 L_{B}+1}}\left(L_{B}+1,1 ; M_{B}+\mu-\nu, \nu \mid L_{B}, M_{B}+\mu\right) \\
& \times\left(L_{B}+1, l: M_{B}+\mu-\nu, 0 \mid L, M_{B}+\mu-\nu\right)\left(L, l ; 0,0 \mid L_{B}+1,0\right) \\
& \times \int f_{L J}(r) j_{l}\left(k_{\gamma} r\right)\left(\frac{d f_{B}(r)}{d r}-L_{B} \frac{f_{B}(r)}{r}\right) r^{2} d r \\
& \mathcal{P}_{L_{B}-1, l, L}^{M_{B}, \mu, \nu}=\sqrt{\frac{L_{B}}{2 L_{B}+1}}\left(L_{B}-1,1 ; M_{B}+\mu-\nu, \nu \mid L_{B}, M_{B}+\mu\right) \\
& \times\left(L_{B}-1, l: M_{B}+\mu-\nu, 0 \mid L, M_{B}+\mu-\nu\right) \\
& \times\left(L, l ; 0,0 \mid L_{B}-1,0\right) \\
& \times \int f_{L J}(r) j_{l}\left(k_{\gamma} r\right)\left(\frac{d f_{B}(r)}{d r}+\frac{L_{B}+1}{r} f_{B}(r)\right) r^{2} d r \\
& H_{L, J, L_{B}}^{M_{B}, \mu, \nu}=\left(L, 1 / 2 ; M_{B}-\mu-\nu, \mu \mid J, M_{B}-\nu\right) \\
& \times\left(L_{B}, 1 / 2 ; M_{B}-\mu, \mu \mid J_{B}, M_{B}\right) \text {. } \\
& \mathcal{H}_{L, J, L_{B}}^{M_{B}, \mu}=\left(L, 1 / 2 ; M_{B}+\mu, \mu \mid J, M_{B}+2 \mu\right) \\
& \times\left(L_{B}, 1 / 2 ; M_{B}+\mu,-\mu \mid J_{B}, M_{B}\right), \\
& C_{l, L, J, L_{B}}^{\mu}=\left(L_{B}, 1 / 2 ; M_{B}+\mu,-\mu \mid J_{B}, M_{B}\right) \\
& \times\left(L, 1 / 2 ; M_{B}+\mu, \mu \mid J, M_{B}+2 \mu\right) \\
& \times\left(L, l ; M_{B}+\mu, 0 \mid L_{B}, M_{B}+\mu\right),
\end{aligned}
$$




\section{Figure Captions}

Fig. 1: Differential cross section (a), and photon asymmetry (b) for the reaction $\left.{ }^{16} O(\gamma, p)\right)^{15} N$ at $E_{\gamma}=100 \mathrm{MeV}$.

Solid curve - full relativistic calculations.

Dotted curve - nonrelativistic calculations using the first order Hamiltonian $H_{I}^{(1)}$ of equation (19).

Dot-dashed curve - second order nonrelativistic calculations (neglecting the nuclear potentials in $H_{I}^{(2)}$ from equation (19)). These are referred to as medium-uncorrected second order calculations in the text.

Dashed curve - second order nonrelativistic calculations using the full $H_{I}^{(2)}$. These are referred to as medium-corrected second order calculations.

Fig. 2: Differential cross section (a), and photon asymmetry (b) for the reaction of Fig. 1 but with $E_{\gamma}=312 \mathrm{MeV}$. Curves labelled as in Fig. 1.

Fig. 3: Differential cross section (a), and photon asymmetry (b) for the reaction ${ }^{16} O(\gamma, p){ }^{15} N$ at $E_{\gamma}=100 \mathrm{MeV}$.

Solid curve - full relativistic calculations.

Dotted curve - nonrelativistic calculations using the first order FW Hamiltonian $H_{I}^{(1)}$ of equation (38) and nucleon wave functions obtained from equation (39) using the first order and spin-orbit terms of equation (36).

Dot-dashed curve - second order nonrelativistic FW calculations (neglecting the nuclear potentials in $H_{I}^{(2)^{\prime}}$ from equation (43)), and full second order potentials to generate the wave functions. These are referred to as mediumuncorrected second order calculations in the text.

Dashed curve - second order nonrelativistic FW calculations using the full $H_{I}^{(2)^{\prime}}$. These are referred to as medium-corrected second order calculations.

Fig. 4: Differential cross section (a), and photon asymmetry (b) for the reaction of Fig 3 but with $E_{\gamma}=196 \mathrm{MeV}$. Curves labelled as in Fig. 3.

Fig. 5: Differential cross section (a), and photon asymmetry (b) for the reaction of Fig 3 but with $E_{\gamma}=312 \mathrm{MeV}$. Curves labelled as in Fig. 3. 


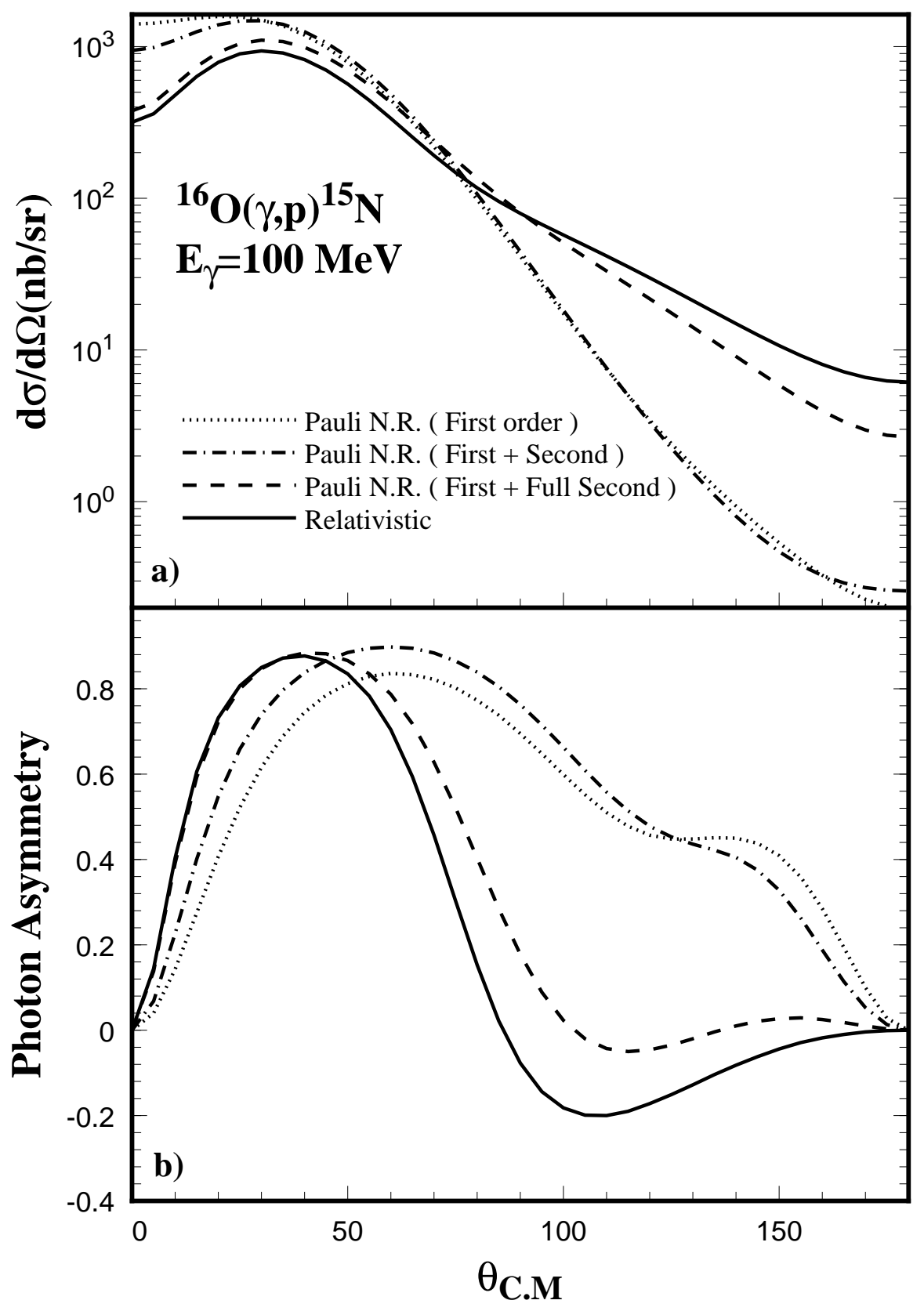

Figure 1 


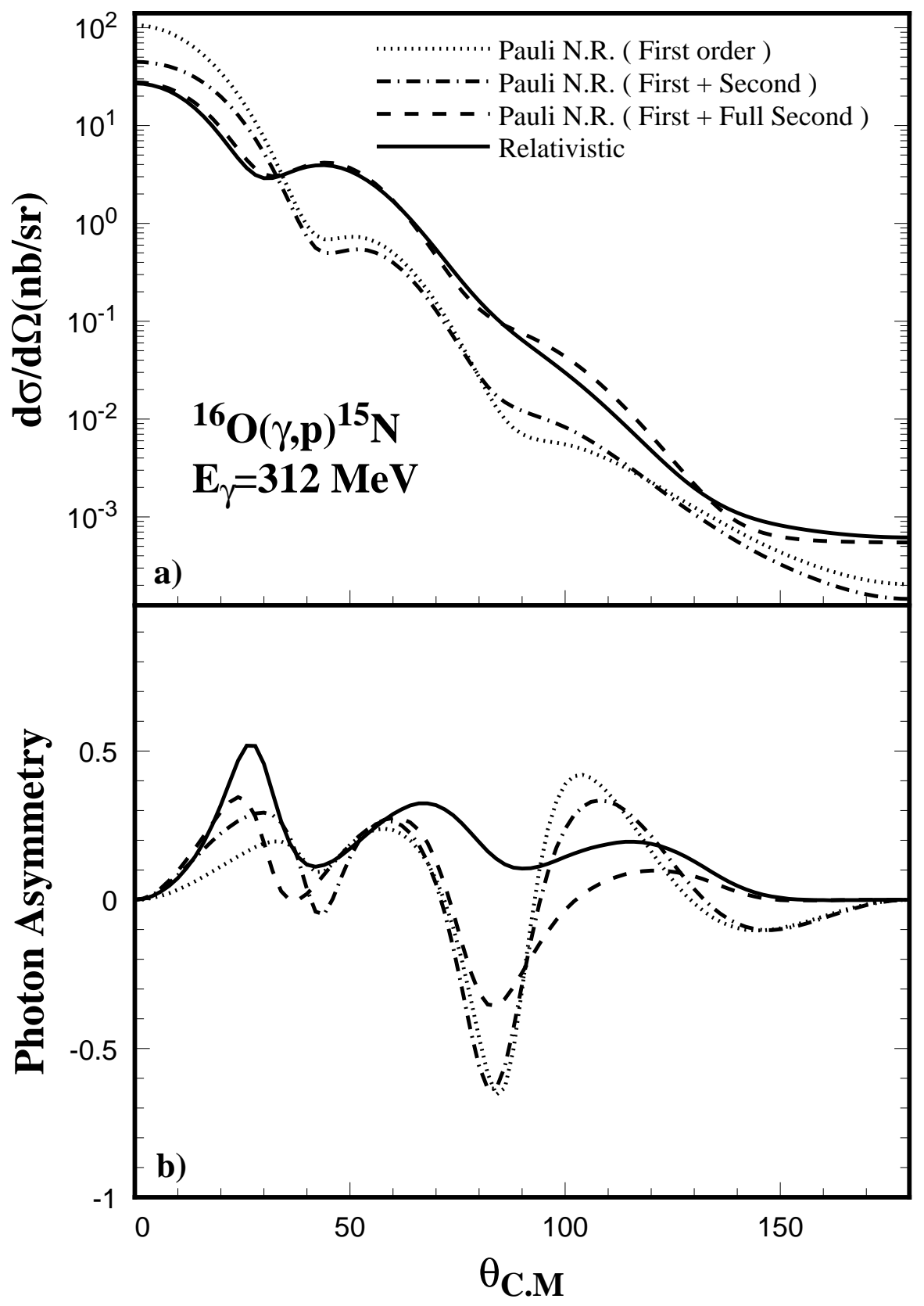

Figure 2 


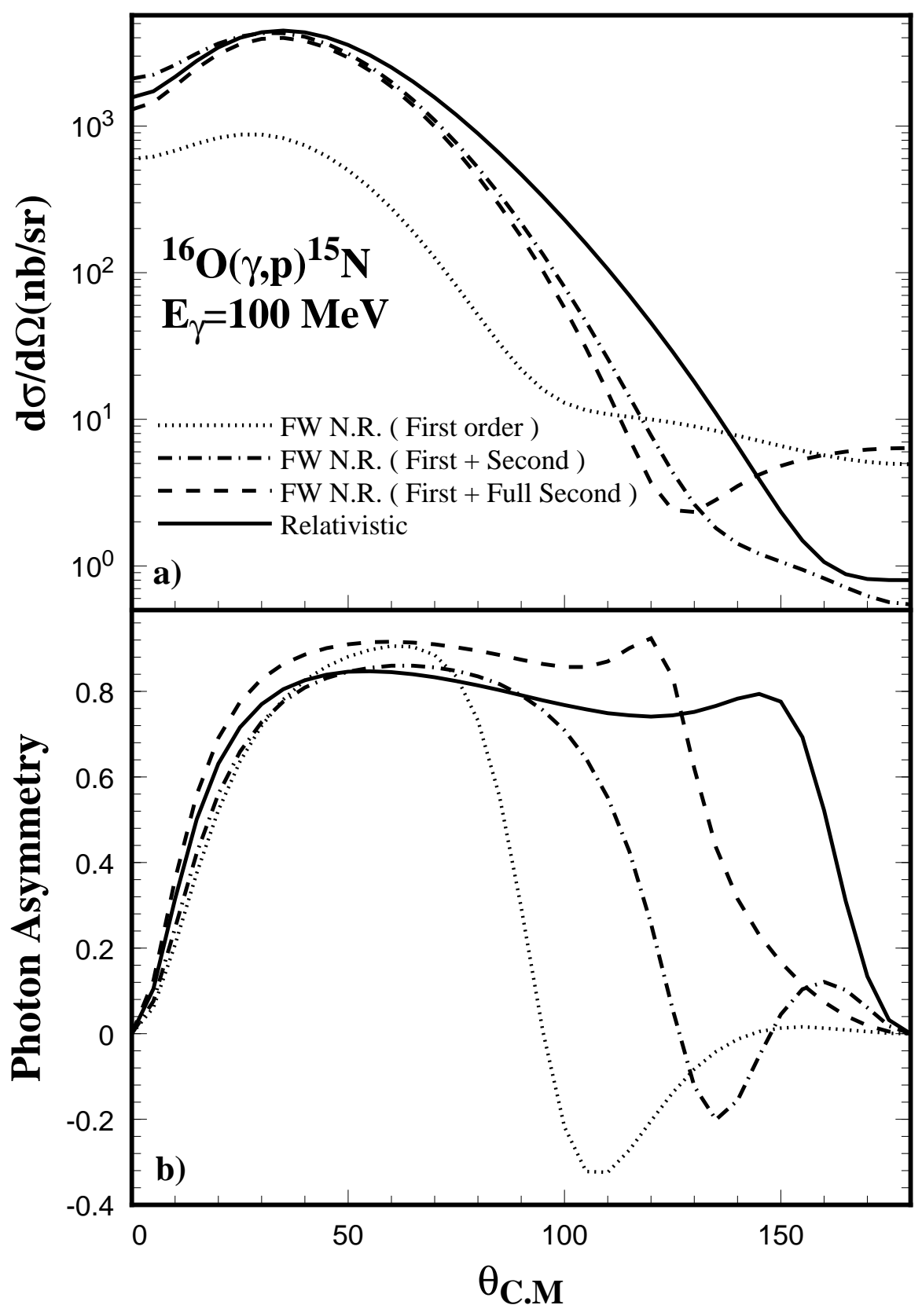

Figure 3 


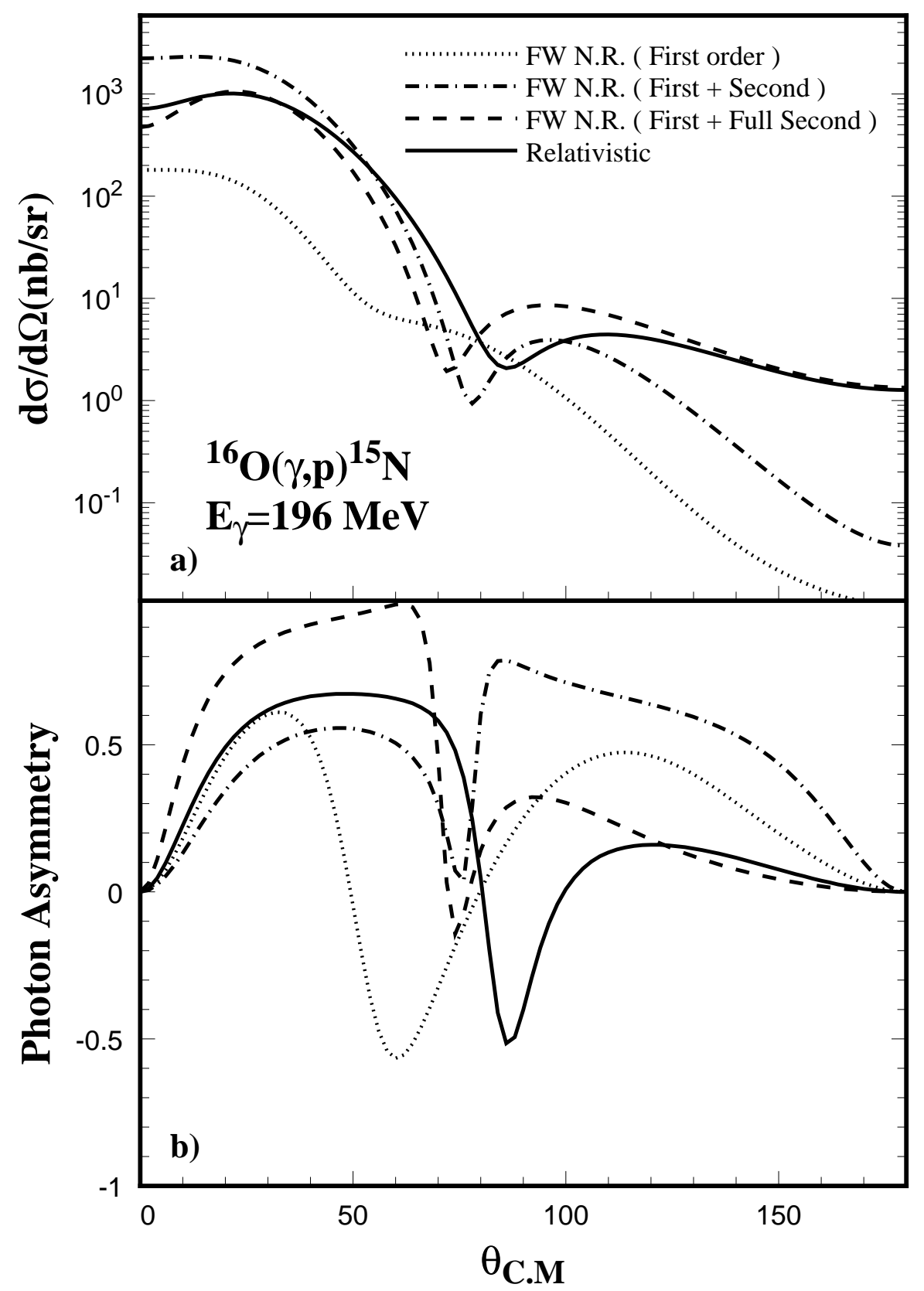

Figure 4 


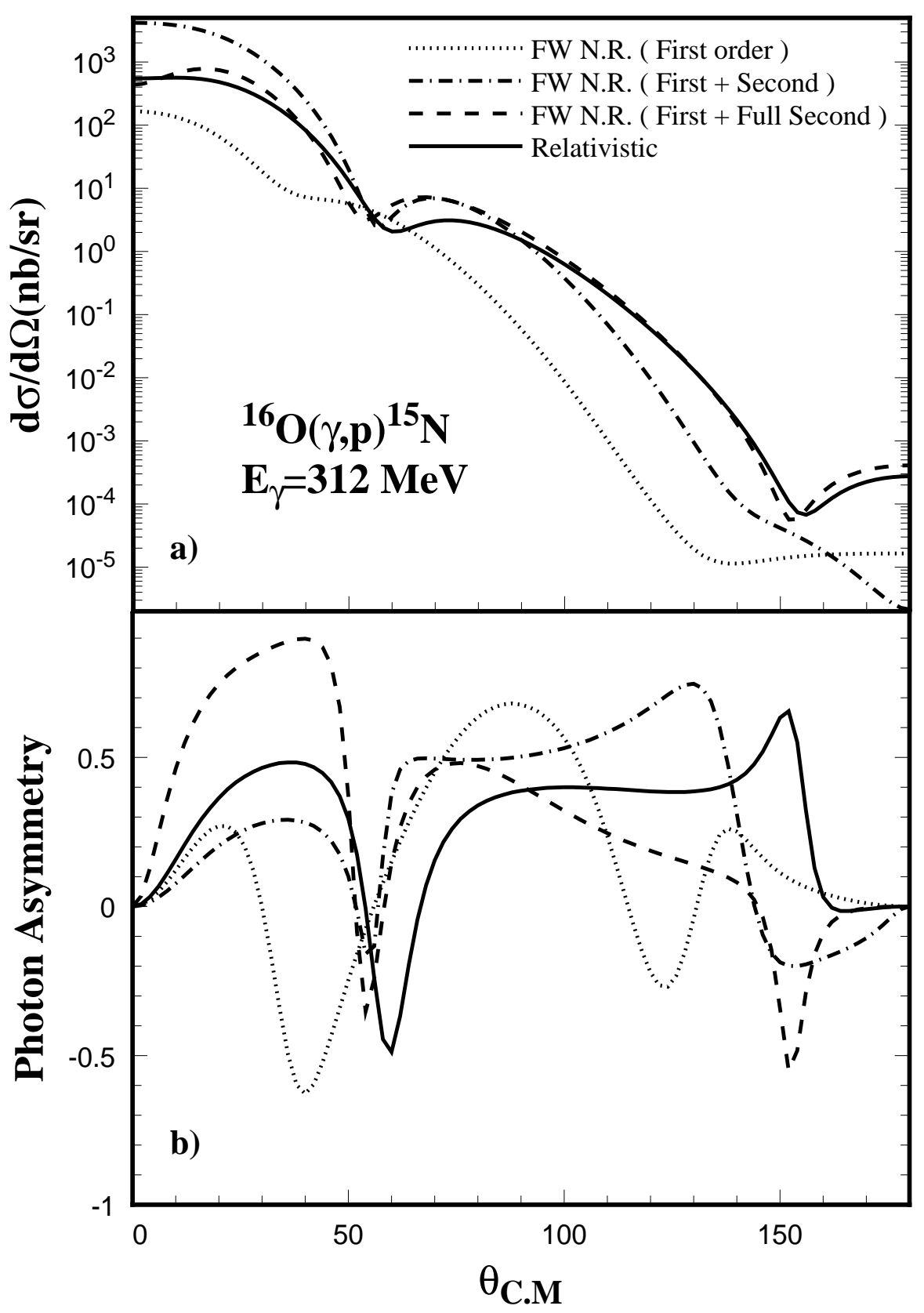

Figure 5 